Document downloaded from:

http://hdl.handle.net/10251/67119

This paper must be cited as:

Barrachina Villalba, J.; Fogue, M.; Garrido, P.; Martínez, FJ.; Cano Escribá, JC.; Tavares De Araujo Cesariny Calafate, CM.; Manzoni, P. (2013). Assessing Vehicular Density Estimation Using Vehicle-to-Infrastructure Communications. 14th IEEE International Symposium on a World of Wireless, Mobile and Multimedia Networks (WoWMoM 2013). IEEE. doi:10.1109/WoWMoM.2013.6583416.

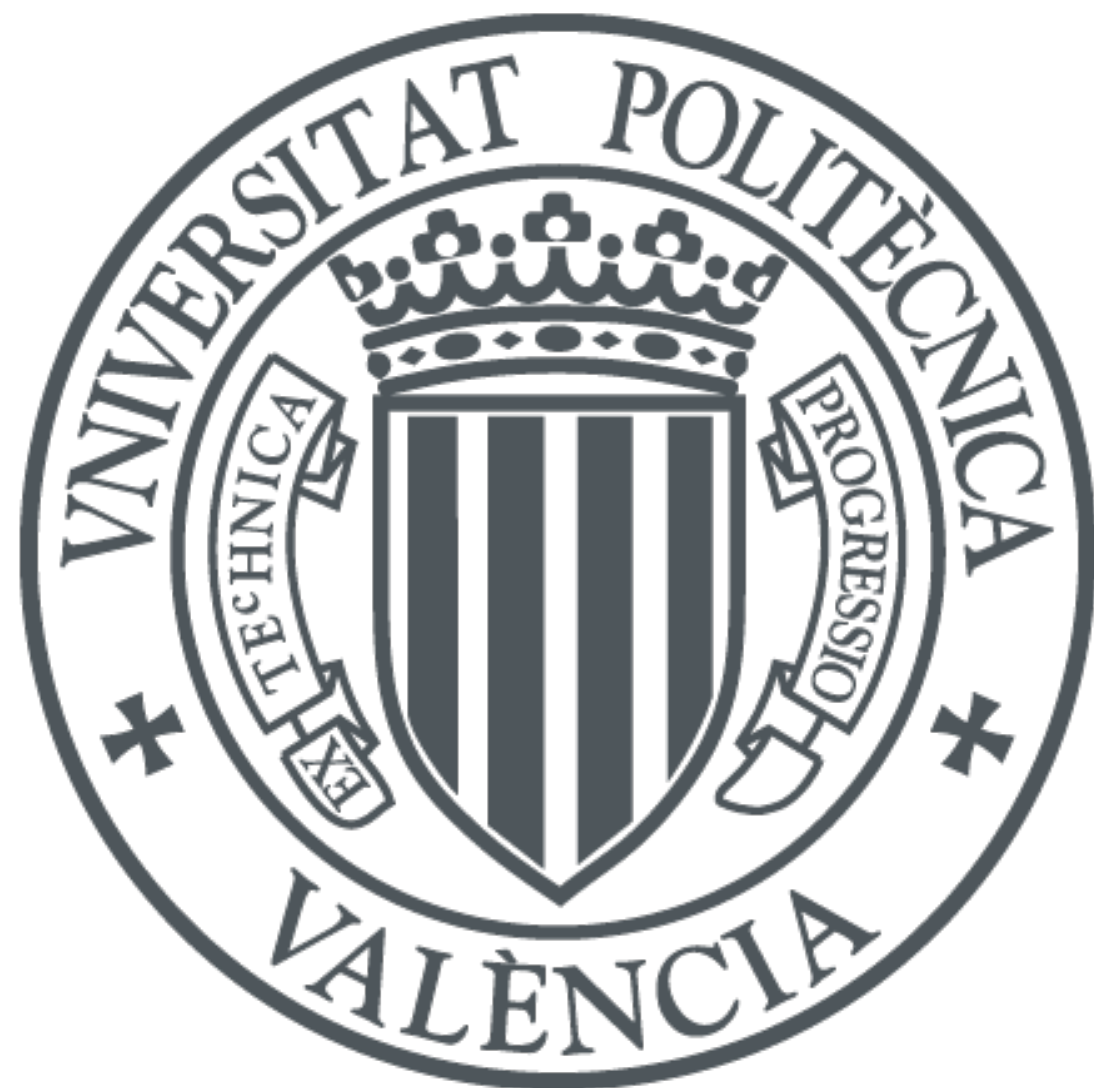

The final publication is available at

http://dx.doi.org/10.1109/WoWMoM.2013.6583416

Copyright IEEE

Additional Information

C2013 IEEE. Personal use of this material is permitted. Permission from IEEE must be obtained for all other uses, in any current or future media, including reprinting/republishing this material for advertising or promotional purposes, creating new collective works, for resale or redistribution to servers or lists, or reuse of any copyrighted component of this work in other works. 


\section{Assessing Vehicular Density Estimation Using Vehicle-to-Infrastructure Communications}

\author{
Javier Barrachina, Manuel Fogue, Piedad Garrido, \\ Francisco J. Martinez \\ Computer Science and System Engineering Department \\ University of Zaragoza, Spain \\ Email: \{barrachina, mfogue, piedad, f.martinez\}@unizar.es
}

\author{
Juan-Carlos Cano, Carlos T. Calafate, Pietro Manzoni, \\ Computer Engineering Department \\ Universitat Politècnica de València, Spain \\ Email: \{jucano, calafate, pmanzoni\}@disca.upv.es
}

\begin{abstract}
Vehicle density is one of the main metrics used for assessing the road traffic conditions. In this paper, we present a solution to estimate the density of vehicles that has been specially designed for Vehicular Networks. Our proposal allows Intelligent Transportation Systems to continuously estimate the vehicular density by accounting for the number of beacons received per Road Side Unit, as well as the roadmap topology. Simulation results indicate that our approach accurately estimates the vehicular density, and therefore automatic traffic controlling systems may use it to predict traffic jams and introduce countermeasures.

Index Terms-Vehicular Networks, vehicular density estimation, Road Side Unit, VANETs.
\end{abstract}

\section{INTRODUCTION}

Traditionally, vehicle density has been one of the main metrics used for assessing the road traffic conditions. A high vehicle density usually indicates that the traffic is congested. However, the density of vehicles circulating in a city highly varies depending on the area and the time during the day.

Currently, most of the vehicle density estimation approaches are designed for using infrastructure-based traffic information systems, which require the deployment of vehicle detecting devices such as inductive loop detectors or traffic surveillance cameras [1], [2]. However, these approaches are limited since they can only be aware of traffic density in a very specific and reduced area (i.e., the streets and junctions in which these devices are already located), making it difficult to estimate the vehicular density of a neighborhood, or a whole city. In addition, some of these approaches are not able to perform the density estimation process in real time (e.g., using cameras involves hard image treatment and analysis).

In this work, we present a solution to estimate the traffic density on the roads that relies on the V2I communication capabilities offered by Vehicular Networks. Unlike previous works, our proposal allows ITS to continuously estimate the vehicular density in a certain area by accounting for the number of beacons received per RSU, as well as the roadmap topology.

The rest of this paper is organized as follows: Section II details our proposal for real-time RSU-based vehicular density estimation, assessing its goodness. Additionally, we discuss the obtained results and measure the estimated error. Finally, Section III concludes this paper.

\section{Real-Time Vehicular Density Estimation}

In this work we propose a method able to accurately estimate the density of vehicles, which is based on the number of beacons received by RSUs and the roadmap topology. We made a total of 900 experiments. These experiments involved the simulation of controlled scenarios (i.e., scenarios where the actual density is known). According to the results obtained, and using a regression analysis, we propose a density estimation function capable of estimating the vehicular density in every urban environment at any instant of time.

\section{A. Features of the Cities Studied}

The roadmaps used during the experiments to achieve the density estimation were selected in order to have different profile scenarios (i.e., with different topology characteristics).

The first step before starting the simulations was to obtain the main features of each roadmap (i.e., the number of streets, the number of junctions, the average distance of segments, and the number of lanes per street). As for the streets, we realized that different alternatives could be selected to obtain the number of streets of a given roadmap. Basically, they are: (i) the number of streets obtained in SUMO [3], where each segment between two junctions is considered a street, (ii) the number of streets obtained in OpenStreetMap (OSM), where each street has a different "name", and (iii) the number of streets according to our Real Attenuation and Visibility (RAV) radio propagation model, where the visibility between vehicles is taken into account when identifying the streets [4].

Table I shows the values obtained according to each criterion to count the number of streets for the cities studied. As shown, the differences between these approaches are significant (e.g., New York has 700, 827, or 257 streets when considering SUMO segments, OSM streets, or the RAV visibility approach, respectively, whereas Sydney has 1668, 315 , or 872 streets, depending on the selected criterion). Therefore, it is important to decide which one to use in order to obtain accurate results. After some experiments, we realized that the third approach better correlated with the real features of cities, since the other two present some drawbacks: they are not accurate enough, or they present some errors. So, we choose this approach for the analysis that follows. 
TABLE II

MAP FEATURES

\begin{tabular}{|c|c|c|c|c|c|}
\hline Map & Streets & Junctions & avg. segment distance (m.) & lanes/street & SJ Ratio \\
\hline New York & 257 & 500 & 45.8853 & 1.0590 & 0.5140 \\
\hline Minnesota & 459 & 591 & 102.0652 & 1.0144 & 0.7766 \\
\hline Madrid & 628 & 715 & 83.0820 & 1.2696 & 0.8783 \\
\hline San Francisco & 725 & 818 & 72.7065 & 1.1749 & 0.8863 \\
\hline Amsterdam & 1494 & 1449 & 44.8973 & 1.1145 & 1.0311 \\
\hline Sydney & 872 & 814 & 72.1813 & 1.2014 & 1.0713 \\
\hline Liverpool & 1758 & 1502 & 49.9620 & 1.2295 & 1.1704 \\
\hline Valencia & 2829 & 2233 & 33.3653 & 1.0854 & 1.2669 \\
\hline Rome & 1655 & 1193 & 45.8853 & 1.0590 & 1.3873 \\
\hline
\end{tabular}

TABLE I

NUMBER OF STREETS OBTAINED DEPENDING ON THE APPROACH USED

\begin{tabular}{|c|c|c|c|}
\hline City & SUMO & OSM & RAV \\
\hline New York & 700 & 827 & 257 \\
\hline Minnesota & 1592 & 105 & 459 \\
\hline Madrid & 1387 & 1029 & 628 \\
\hline San Francisco & 1710 & 606 & 725 \\
\hline Amsterdam & 3022 & 796 & 1494 \\
\hline Sydney & 1668 & 315 & 872 \\
\hline Liverpool & 3141 & 1042 & 1758 \\
\hline Valencia & 5154 & 1050 & 2829 \\
\hline Rome & 2780 & 1484 & 1655 \\
\hline
\end{tabular}

Table II shows the main map features for the cities under study (i.e., the number of streets according to the RAV algorithm, the number of junctions, the average distance of segments, and the number of lanes per street). We also added a column labeled as SJ Ratio, which represents the result of dividing the number of streets between the number of junctions. As shown, the first city (New York) presents an SJ ratio of 0.5130 , which indicates that it has a simple topology, whereas the last cities in the table present a greater value, which indicates a more complex topology. This aggregated factor correlates well with the obtained results.

\section{B. Simulation Environment}

Simulations were done using the ns-2 simulator, where the PHY and MAC layers have been modified to closely follow the IEEE $802.11 \mathrm{p}$ standard, which defines enhancements to the 802.11 required to support ITS applications. We assume that all the nodes of our network have two different interfaces: (i) an IEEE $802.11 \mathrm{n}$ interface tuned at the frequency of $2.4 \mathrm{GHz}$ for V2I communications, and (ii) an IEEE 802.11p interface tuned at the frequency of $5 \mathrm{GHz}$ for $\mathrm{V} 2 \mathrm{~V}$ communications. To prove how maps affect the performance of vehicular communications, we selected nine street maps, each one representing a square area of $4 \mathrm{~km}^{2}$. In order to deploy RSUs in the maps, we use the Uniform Mesh deployment policy [5], that consists on distributing RSUs uniformly on the map. The advantage of this deployment policy is that it achieves a more uniform coverage area since the distance between RSUs is the same, preventing RSUs to be positioned too closely, or too sparsely. As for the mobility of the vehicles, it has been performed with CityMob for Roadmaps (C4R) [6], a mobility generator able to import maps directly from OpenStreetMap, and generate ns-2 compatible traces. Table III shows the parameters used.

We tested our proposal by evaluating the performance of
TABLE III

PARAMETERS USED FOR THE SIMULATIONS

\begin{tabular}{|l|c|}
\hline Parameter & Value \\
\hline roadmaps & $\begin{array}{c}\text { New York, Minnesota, Madrid, } \\
\text { San Francisco, Amsterdam, Sydney, } \\
\text { Liverpool, Valencia, and Rome }\end{array}$ \\
roadmap size & $2000 \mathrm{~m} \times 2000 \mathrm{~m}$ \\
number of vehicles & {$[100,200,300 \ldots 1000]$} \\
beacon message size & $512 B$ \\
warning messages priority & $A C 3$ \\
beacon priority & $A C 1$ \\
interval between messages & $1 \mathrm{~second}$ \\
RSU deployment policy & Uniform Mesh [5] \\
MAC/PHY & $802.11 \mathrm{p}$ \\
radio propagation model & RAV [4] \\
mobility model & Krauss [8] \\
channel bandwidth & $6 M b p s$ \\
max. transmission range & $400 \mathrm{~m}$ \\
simulation time & $30 \mathrm{~s}$ \\
\hline
\end{tabular}

a Warning Message Dissemination mechanism, where each vehicle periodically broadcasts information about itself or about an abnormal situation (traffic jams, icy roads, etc.). To increase the realism of our results, we include the possibility that vehicles share accident notification messages in our simulations. In fact, we consider that vehicles can operate in two different modes: (a) warning, and (b) normal. Vehicles in warning mode inform other vehicles about their status by sending warning messages periodically (every second). Normal mode vehicles enable the diffusion of these warning packets and, every second, they also send beacons with information such as their positions, speed, etc. These periodic messages are not propagated by other vehicles. The warning messages exchanged between vehicles and RSUs are built according to the Vehicular Accident Ontology (VEACON) [7], which provides a standard structure to enable data interoperability among the different entities involved in transportation systems.

\section{Density Estimation Function}

After performing the topological analysis of the studied maps, we obtained the number of beacons received by each RSU during 30 seconds, taking into account that each vehicle sends one beacon per second, and that these messages, unlike warning messages, are not disseminated by the rest of the vehicles. Figure 1 shows the results obtained for the different cities studied. As shown, the performance in New York and Minnesota in terms of number of beacons received highly differs from the rest of the cities. This is caused because New 


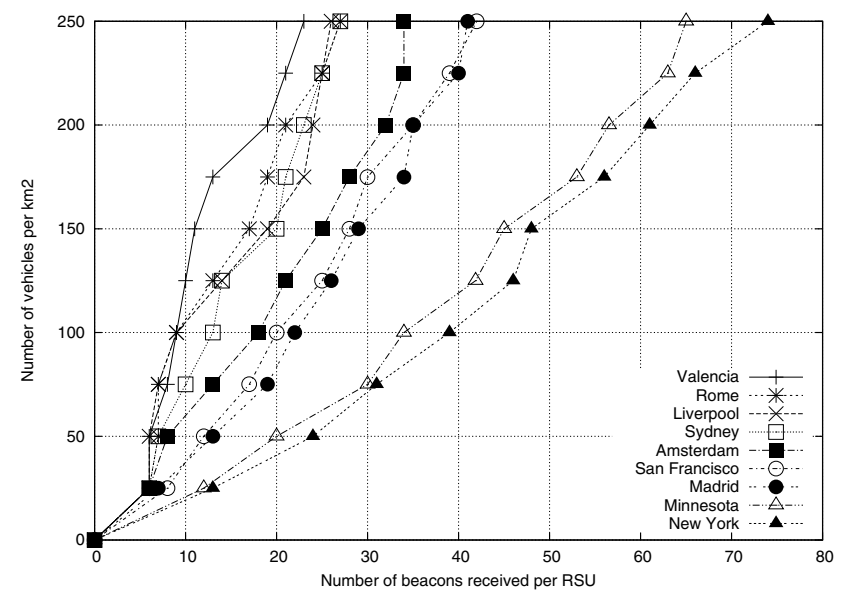

Fig. 1. Number of beacons received when varying the vehicular density.

TABLE IV

PROPOSED EQUATION COEFFICIENTS

\begin{tabular}{|c|c|}
\hline Coeff. & Value \\
\hline a & $2.4328753582642619 \mathrm{E}+02$ \\
\hline b & $8.8667060945557523 \mathrm{E}+00$ \\
\hline c & $-4.2340086242746855 \mathrm{E}+02$ \\
\hline d & $3.2563178030488615 \mathrm{E}+01$ \\
\hline f & $1.8200236614892370 \mathrm{E}+02$ \\
\hline g & $-6.4626326366022894 \mathrm{E}+01$ \\
\hline
\end{tabular}

York and Minnesota have a low SJ ratio (i.e., they are simple roadmaps). As expected, complex roadmaps (maps which have a higher SJ Ratio) present a number of received beacons lower than simple roadmaps for a similar vehicular density. Figure 1 also shows that the vehicular density not only depends on the number of beacons received, but also on the SJ ratio (according to data shown in Table II). Therefore, the characteristics of the roadmap will be very useful in order to accurately estimate the vehicular density in a given scenario.

After observing the direct relationship between the topology of the maps, the number of beacons received, and the density of vehicles, we proceed to obtain a function to estimate, with the minimum possible error, each of the curves shown in Figure 1. To this purpose, we performed a regression analysis that allowed us to find an algebraic expression offering the best fit to the data obtained through simulation.

Equation 1 shows the density estimation function, which is able to estimate the number of vehicles per $\mathrm{km}^{2}$ in urban scenarios, according to the number of beacons received per RSU, and the SJ ratio (i.e., streets/junctions).

$f(x, y)=a+b \cdot \ln (x)+\frac{c}{y}+d \cdot \ln (x)^{2}+\frac{f}{y^{2}}+\frac{g \cdot \ln (x)}{y}$

In this equation, $x$ is the number of beacons received by each RSU, and $y$ is the SJ ratio obtained from the roadmap. The values of the polynomial coefficients $(a, b, c, d, f$, and $g)$ are listed in Table IV.

To determine the accuracy of our proposal, it is necessary to measure the estimated error. Table $\mathrm{V}$ shows the different types of errors calculated when comparing our density estimation
TABLE V

DENSITY ESTIMATION ERROR

\begin{tabular}{|c|c|c|}
\hline Error & Absolute & Relative \\
\hline Minimum & $-5.736426 \mathrm{E}+01$ & $-1.218902 \mathrm{E}+00$ \\
\hline Maximum & $5.135632 \mathrm{E}+01$ & $1.784647 \mathrm{E}+00$ \\
\hline Mean & $-1.642143 \mathrm{E}-14$ & $3.634060 \mathrm{E}-02$ \\
\hline Std. Error of Mean & $2.596603 \mathrm{E}+00$ & $3.592458 \mathrm{E}-02$ \\
\hline Median & $-1.914503 \mathrm{E}+00$ & $-2.313015 \mathrm{E}-02$ \\
\hline
\end{tabular}

function with the values actually obtained. Note that the average relative error is of only $3.63 \%$. We consider that this error can be neglected, thus validating our proposed function.

\section{Conclusions}

This paper proposes a method that allows estimating the vehicular density in urban environments at any given time by using V2I communications. To develop the vehicular density estimation algorithm, we have taken into account not only the number of beacons received by the RSUs, but also the topology of the map where the vehicles are located. As a result of a large number of simulations, we have obtained an equation that is able to accurately predict the vehicular density. Results show that our proposal allows estimating the vehicular density for any given city with a high accuracy, thereby allowing governments to improve their traffic control mechanisms.

\section{ACKNOWLEDGMENTS}

This work was partially supported by the Ministerio de Ciencia e Innovación, Spain, under Grant TIN2011-27543$\mathrm{C} 03-01$.

\section{REFERENCES}

[1] V. Tyagi, S. Kalyanaraman, and R. Krishnapuram, "Vehicular traffic density state estimation based on cumulative road acoustics," IEEE Transactions on Intelligent Transportation Systems, vol. 13, no. 3, pp. 1156-1166, Sept. 2012.

[2] E. Tan and J. Chen, "Vehicular traffic density estimation via statistical methods with automated state learning," in IEEE Conference on Advanced Video and Signal Based Surveillance (AVSS), Sept. 2007, pp. 164-169.

[3] D. Krajzewicz and C. Rossel, "Simulation of Urban MObility (SUMO)," Centre for Applied Informatics (ZAIK) and the Institute of Transport Research at the German Aerospace Centre, 2012, Available at http://sumo.sourceforge.net.

[4] M. Fogue, P. Garrido, F. J. Martinez, J.-C. Cano, C. T. Calafate, and P. Manzoni, "Evaluating the impact of a novel message dissemination scheme for vehicular networks using real maps," Transportation Research Part C: Emerging Technologies, vol. 25, pp. 61-80, Dec. 2012. [Online]. Available: http://dx.doi.org/10.1016/j.trc.2012.04.017

[5] J. Barrachina, P. Garrido, M. Fogue, F. J. Martinez, J.-C. Cano, C. T. Calafate, and P. Manzoni, "D-RSU: A Density-Based Approach for Road Side Unit Deployment in Urban Scenarios," in International Workshop on IPv6-based Vehicular Networks (Vehi6), collocated with the 2012 IEEE Intelligent Vehicles Symposium, Jun. 2012, pp. 1-6.

[6] M. Fogue, P. Garrido, F. J. Martinez, J.-C. Cano, C. T. Calafate, and P. Manzoni, "A Realistic Simulation Framework for Vehicular Networks," in 5th International ICST Conference on Simulation Tools and Techniques (SIMUTools 2012), Desenzano, Italy, Mar. 2012, pp. 37-46.

[7] J. Barrachina, P. Garrido, M. Fogue, F. J. Martinez, J.-C. Cano, C. T. Calafate, and P. Manzoni, "VEACON: A Vehicular Accident Ontology designed to improve safety on the roads," Journal of Network and Computer Applications, vol. 35, no. 6, pp. 1891-1900, Nov. 2012. [Online]. Available: http://dx.doi.org/10.1016/j.jnca.2012.07.013

[8] S. Krauss, P. Wagner, and C. Gawron, "Metastable states in a microscopic model of traffic flow," Physical Review E, vol. 55, no. 5, pp. 5597-5602, 1997. 